J. Nonlinear Var. Anal. 2 (2018), No. 3, pp. 273-286

Available online at http://jnva.biemdas.com

https://doi.org/10.23952/jnva.2.2018.3.03

\title{
ALGORITHMS FOR SPLIT COMMON FIXED POINT PROBLEMS IN HILBERT SPACES
}

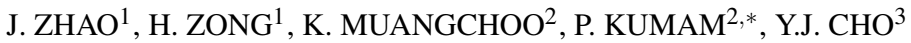 \\ ${ }^{1}$ College of Science, Civil Aviation University of China, Tianjin 300300, China \\ ${ }^{2}$ KMUTT Fixed Point Research Laboratory, Department of Mathematics, \\ Room SCL 802 Fixed Point Laboratory, Science Laboratory Building, Faculty of Science, \\ King Mongkut's University of Technology Thonburi (KMUTT), \\ 126 Pracha-Uthit Road, Bang Mod, Thrung Khru, Bangkok 10140, Thailand \\ ${ }^{3}$ Department of Mathematics Education and RINS, Gyeongsang National University, Jinju 660-701, Korea
}

\begin{abstract}
In this paper, we use the dual variable to propose two algorithms for split common fixed-point problems of averaged mappings in real Hilbert spaces. Under suitable conditions, weak and strong convergence theorems are established. Finally, we give numerical experiments to illustrate the efficiency of the proposed iterative algorithms.

Keywords. Split common fixed-point problem; Variational inequality problem; Iterative algorithm; Dual variable; Averaged mapping.
\end{abstract}

2010 Mathematics Subject Classification. 47H09, 47N10.

\section{INTRODUCTION}

In 2009, Censor and Segal [4] introduced a split common fixed point problem, which is an inverse problem, aiming to find an element in a fixed point set such that its image under a bounded linear operator belongs to another fixed point set, in finite dimensional Hilbert spaces. Recently, many authors investigated the split common fixed point problem via iterative methods in difference spaces.

Let $H_{1}$ and $H_{2}$ be two real Hilbert spaces. The Split Common Fixed Point problem (in short, SCFP) can be formulated as the problem of finding

$$
x^{*} \in F(U) \text { such that } A x^{*} \in F(T),
$$

where $A: H_{1} \rightarrow H_{2}$ is a bounded linear operator, $F(U)$ and $F(T)$ stand for the fixed point sets of $U$ : $H_{1} \rightarrow H_{1}$ and $T: H_{2} \rightarrow H_{2}$, respectively. In particular, if $U$ and $T$ are projection operators, then the SCFP is reduced to the known split feasibility problem (shortly, SFP) [3,5], which is to find a point $x^{*}$ such that

$$
x^{*} \in C \text { such that } A x^{*} \in Q,
$$

where $C$ and $Q$ are nonempty closed convex subsets of $H_{1}$ and $H_{2}$, respectively. Such problems arise in the field of intensity-modulated radiation therapy when one attempts to describe physical dose constraints

\footnotetext{
${ }^{*}$ Corresponding author.

E-mail addresses: zhaojing200103@163.com (J. Zhao), 861350555@qq.com (H. Zong), ni_003@hotmail.com (K. Muangchoo), poom.kumam@mail.kmutt.ac.th (P. Kumam), yjcho@gnu.ac.kr (Y.J. Cho).

Received January 4, 2018; Accepted March 16, 2018.
}

(C)2018 Journal of Nonlinear and Variational Analysis 
and equivalent uniform dose constraints within a single model (see [6]). Since the split feasibility problem in finite dimensional Hilbert spaces was first introduced by Censor and Elfving [5], many algorithms have been introduced to solve the SFP; see $[2,7,18,13]$ and references therein.

Note that, if (1.2) is consistent (i.e., (ref1.2) has a solution), it is no hard to see that $x^{*}$ solves (1.2) if and only if it solves the fixed point equation:

$$
P_{C}\left(I-\gamma A^{*}\left(I-P_{Q}\right) A\right) x^{*}=x^{*},
$$

where $P_{C}$ and $P_{Q}$ are the (orthogonal) projection onto $C$ and $Q$, respectively, $\gamma>0$ is a positive constant and $A^{*}$ denotes the adjoint of $A$. This implies that we can use fixed point algorithms to solve the SFP.

To solve SFP (1.2), Byrne [3] proposed the CQ-algorithm which generates a sequence $\left\{x_{k}\right\}$ by

$$
x_{k+1}=P_{C}\left(I-\gamma A^{*}\left(I-P_{Q}\right) A\right) x_{k}
$$

for each $k \in \mathbb{N}$, where $\gamma \in\left(0, \frac{2}{\lambda}\right)$ with $\lambda$ being the spectral radius of $A^{*} A$. For solving SCFP (1.1), Censor and Segal [4] proposed and proved, in finite dimensional spaces, the convergence of the following iterative scheme:

$$
x_{n+1}=U\left(x_{n}-\gamma A^{*}(I-T) A x_{n}\right),
$$

where $\gamma$ is chosen in the interval $\left(0, \frac{2}{\lambda}\right)$ with $\lambda$ being the spectral radius of $A^{t} A$, where $A^{t} A$ stands for matrix transposition. Recently many authors have introduced some algorithms to solve the SCFP (1.1); see $[8,9,15,16,17,19]$ and the references therein.

In this paper, we use the dual variable to propose two algorithms for the SCFP governed by averaged mappings. In [10], Chen, Huang and Zhang considered to minimize the sum of two proper lower semicontinuous convex functions, i.e.,

$$
x^{*}=\arg \min _{x \in \mathbb{R}^{n}} f_{1}(x)+f_{2}(x),
$$

where $f_{1}, f_{2} \in \Gamma_{0}\left(\mathbb{R}^{n}\right)$ (: the set of all proper lower semi-continuous convex functions from $\mathbb{R}^{n}$ to $(-\infty,+\infty])$ and $f_{2}$ is differentiable on $R^{n}$ with $\frac{1}{\beta}$-Lipschitz continuous gradient for some $\beta \in(0,+\infty)$. To solve convex separable problem (1.6), they obtained the following fixed point formulation: the point $x^{*}$ is a solution of problem (1.6) if and only if there exists $v^{*} \in \mathbb{R}^{m}$ such that

$$
\left\{\begin{array}{l}
v^{*}=\left(I-\operatorname{prox}_{\frac{\gamma}{\lambda} f_{1}}\right)\left(x^{*}-\gamma \nabla f_{2}\left(x^{*}\right)+(1-\lambda) v^{*}\right), \\
x^{*}=x^{*}-\gamma \nabla f_{2}(x)-\lambda v^{*}
\end{array}\right.
$$

where $\lambda$ and $\gamma$ are two positive numbers. They introduced the following Picard sequence:

$$
\left\{\begin{array}{l}
v_{k+1}=\left(I-\operatorname{prox}_{\frac{\gamma}{\lambda} f_{1}}\right)\left(x_{k}-\gamma \nabla f_{2}\left(x_{k}\right)+(1-\lambda) v_{k}\right), \\
x_{k+1}=x_{k}-\gamma \nabla f_{2}\left(x_{k}\right)-\lambda v_{k+1} .
\end{array}\right.
$$

It was shown [10] that, under appropriate conditions, sequence $\left\{x_{k}\right\}$ converges to a solution of problem (1.6). Since $x$ is the primal variable related to (1.6), it is very natural to ask what role the variable $v$ plays in above algorithm. After thorough study, they found out that $v$ is actually the dual variable of the primal-dual form related to problem (1.6).

Inspired and motivated by the works mentioned above, for solving SCFP (1.1) of averaged mappings, we use the dual variable to propose a weak convergence algorithm and a strong convergence algorithm modified by the viscosity approximation method. The contents of this paper are as follows. In Section 
2, we give some useful definitions and results for the convergence analysis of the iterative algorithms. In Section 3, we prove some weak convergence theorems of the proposed algorithm with the dual variable. In Section 4, we prove some strong convergence theorems of the modified algorithm. Finally, we give some numerical experiments to illustrate the efficiency of the proposed iterative methods in Section 5.

\section{PReliminaries}

In this paper, we denote the inner product by $\langle\cdot, \cdot\rangle$ and the norm by $\|\cdot\|$. We use $\rightarrow$ and $\rightarrow$ to denote the strong convergence and weak convergence, respectively. We use $\omega_{w}\left(x_{k}\right)=\left\{x: \exists x_{k_{j}} \rightarrow x\right\}$ stand for the weak $\omega$-limit set of $\left\{x_{k}\right\}$.

Definition 2.1. A mapping $T: H \rightarrow H$ is said to be nonexpansive if

$$
\|T x-T y\| \leq\|x-y\|
$$

for all $x, y \in H$.

Definition 2.2. A mapping $T: H \rightarrow H$ is said to be firmly nonexpansive if $2 T-I$ is nonexpansive or, equivalently,

$$
\langle x-y, T x-T y\rangle \geq\|T x-T y\|^{2}
$$

for all $x, y \in H$. A mapping $T: H \rightarrow H$ is said to be firmly quasi-nonexpansive (also directed) if $F(T) \neq \emptyset$ and

$$
\langle x-q, T x-q\rangle \geq\|T x-q\|^{2}
$$

for all $x \in H$ and $q \in F(T)$.

Alternatively, a mapping $T: H \rightarrow H$ is firmly nonexpansive if and only if $T$ can be expressed as

$$
T=\frac{1}{2}(I+S)
$$

where $I$ denotes the identity mapping on $H$ and $S: H \rightarrow H$ is a nonexpansive mapping.

Definition 2.3. A mapping $T: H \rightarrow H$ is said to be an averaged mapping if it can be written as the average of the identity $I$ and a nonexpansive operator, that is,

$$
T=(1-\alpha) I+\alpha S
$$

where $\alpha$ is a number in $(0,1)$ and $S: H \rightarrow H$ is nonexpansive. More precisely, when the above equality holds, we say that $T$ is $\alpha$-averaged.

Definition 2.4. A mapping $T: H \rightarrow H$ is said to be demiclosed at the origin if, for any sequence $\left\{x_{n}\right\}$ which weakly converges to $x$, if the sequence $\left\{T x_{n}\right\}$ strongly converges to 0 , then $T x=0$.

Definition 2.5. A mapping $f: H \rightarrow H$ is said to be $\rho$-contraction if there exists a constant $\rho \in[0,1)$ such that

$$
\|f(x)-f(y)\| \leq \rho\|x-y\|
$$

for all $x, y \in H$. 
Definition 2.6. A mapping $f: C \rightarrow H$ is said to be $\eta$-strongly monotone if there exists a positive constant $\eta$ such that

$$
\langle f(x)-f(y), x-y\rangle \geq \eta\|x-y\|^{2}
$$

for all $x, y \in C$.

It is obvious that, if $f$ is a $\rho$-contraction, then $I-f$ is a $(1-\rho)$-strongly monotone mapping.

Recall the classical variational inequality problem is to find a point $x^{*} \in C$ such that

$$
\left\langle F x^{*}, x-x^{*}\right\rangle \geq 0
$$

for all $x \in C$, where $C$ is a nonempty closed convex subset of $H$ and $F: C \rightarrow H$ is a nonlinear operator.

It is well known that if $F: C \rightarrow H$ is a Lipschitzian and strongly monotone operator, then the above variational inequality problem has a unique solution.

Definition 2.7. For any $\alpha>0$, a mapping $T: H \rightarrow H$ is said to be $\alpha$-inverse strongly monotone (shortly, $\alpha$-ism) if

$$
\langle x-y, T x-T y\rangle \geq \alpha\|T x-T y\|^{2}
$$

for all $x, y \in H$.

Proposition 2.1. [2] A mapping $T: H \rightarrow H$ is averaged if and only if the complement $I-T$ is $v$-ism for some $v>\frac{1}{2}$. Indeed, for $\alpha \in(0,1), T$ is $\alpha$-averaged if and only if $I-T$ is $\frac{1}{2 \alpha}$-ism.

Lemma 2.1. [1] Let $E$ be a uniformly convex Banach space, $K$ be a nonempty closed convex subset of $E$ and $T: K \rightarrow K$ be a nonexpansive mapping. Then $I-T$ is demiclosed at the origin.

Lemma 2.2. [11] Let $K$ be a nonempty closed convex subset of real Hilbert space H. Let $\left\{x_{k}\right\}$ be a bounded sequence which satisfies the following properties:

(a) every weak limit point of $\left\{x_{k}\right\}$ lies in $K$;

(b) $\lim _{k \rightarrow \infty}\left\|x_{k}-x\right\|$ exists for every $x \in K$.

Then $\left\{x_{k}\right\}$ converges weakly to a point in $K$.

Lemma 2.3. [12] Assume that $\left\{s_{k}\right\}$ is a sequence of nonnegative real numbers such that

$$
\left\{\begin{array}{l}
s_{k+1} \leq\left(1-\lambda_{k}\right) s_{k}+\lambda_{k} \delta_{k}, \\
s_{k+1} \leq s_{k}-\eta_{k}+\mu_{k}
\end{array}\right.
$$

for each $k \geq 0$, where $\left\{\lambda_{k}\right\}$ is a sequence in $(0,1),\left\{\eta_{k}\right\}$ is a sequence of nonnegative real numbers and $\left\{\delta_{k}\right\}$ and $\left\{\mu_{k}\right\}$ are two sequences in $\mathbb{R}$ such that

(a) $\Sigma_{k=1}^{\infty} \lambda_{k}=\infty$;

(b) $\lim _{k \rightarrow \infty} \mu_{k}=0$;

(c) $\lim _{l \rightarrow \infty} \eta_{k_{l}}=0$ implies $\lim \sup _{l \rightarrow \infty} \delta_{k_{l}} \leq 0$ for any subsequence $\left\{k_{l}\right\} \subset\{k\}$.

Then $\lim _{k \rightarrow \infty} s_{k}=0$.

The following lemma is trivial.

Lemma 2.4. Let $H$ be a real Hilbert space. Then, for all $t \in[0,1]$ and $x, y \in H$,

$$
\|t x+(1-t) y\|^{2}=t\|x\|^{2}+(1-t)\|y\|^{2}-t(1-t)\|x-y\|^{2} .
$$




\section{WEAK CONVERGENCE THEOREMS}

Throughout this paper, we assume that the SCFP (1.1) is consistent and its solution set is denoted by $\Gamma$, i.e.,

$$
\Gamma=\{x \in F(U): A x \in F(T)\} .
$$

Now, we use the dual variable to establish a new iterative algorithm for the SCFP (1.1) of averaged mappings.

Theorem 3.1. Let $U$ be an $\alpha_{1}$-averaged mapping and $T$ be an $\alpha_{2}$-averaged mapping. Suppose that $0<\lambda \leq 1,0<\alpha_{1} \leq \frac{1}{2}$ and

$$
0<\liminf _{k \rightarrow \infty} \gamma_{k} \leq \limsup _{k \rightarrow \infty} \gamma_{k}<\frac{1}{\alpha_{2}\|A\|^{2}}
$$

Let $\left\{\left(v_{k}, x_{k}\right)\right\}$ be the sequence generated by

$$
\left\{\begin{array}{l}
x_{0}, v_{0} \in H_{1} \text { chosen arbitrarily } \\
y_{k}=x_{k}-\gamma_{k} A^{*}(I-T) A x_{k} \\
v_{k+1}=(I-U)\left(y_{k}+(1-\lambda) v_{k}\right) \\
x_{k+1}=y_{k}-\lambda v_{k+1}
\end{array}\right.
$$

for each $k \geq 0$, then the sequence $\left\{x_{k}\right\}$ converges weakly to a point $x^{*}$, where $x^{*} \in \Gamma$, and the sequence $\left\{\left(v_{k}, x_{k}\right)\right\}$ weakly converges to the point $\left(0, x^{*}\right)$.

Proof. First, we show that $\lim _{k \rightarrow \infty}\left\|x_{k}-x^{*}\right\|$ exists for any $x^{*} \in \Gamma$. Taking $x^{*} \in \Gamma$, we have $x^{*} \in F(U)$ and $A x^{*} \in F(T)$. Since $U$ and $T$ are $\alpha_{1}$-averaged mappings and $\alpha_{2}$-averaged mapping, respectively, by Proposition 2.1, it follows that $I-U$ and $I-T$ are $\frac{1}{2 \alpha_{1}}$-ism and $\frac{1}{2 \alpha_{2}}$-ism, respectively. Thus, from the iterative algorithm (3.1), we have

$$
\begin{aligned}
\left\|v_{k+1}\right\|^{2} & =\left\|(I-U)\left(y_{k}+(1-\lambda) v_{k}\right)-(I-U) x^{*}\right\|^{2} \\
& \leq 2 \alpha_{1}\left\langle v_{k+1}, y_{k}-x^{*}+(1-\lambda) v_{k}\right\rangle
\end{aligned}
$$

and

$$
\begin{aligned}
\left\|x_{k+1}-x^{*}\right\|^{2} & =\left\|y_{k}-\lambda v_{k+1}-x^{*}\right\|^{2} \\
& =\left\|y_{k}-x^{*}\right\|^{2}-2 \lambda\left\langle y_{k}-x^{*}, v_{k+1}\right\rangle+\lambda^{2}\left\|v_{k+1}\right\|^{2} .
\end{aligned}
$$

It follows that

$$
\begin{aligned}
& \left\|x_{k+1}-x^{*}\right\|^{2}+\lambda\left\|v_{k+1}\right\|^{2} \\
= & \left\|y_{k}-x^{*}\right\|^{2}-2 \lambda\left\langle y_{k}-x^{*}, v_{k+1}\right\rangle+\lambda^{2}\left\|v_{k+1}\right\|^{2}+\lambda\left\|v_{k+1}\right\|^{2} \\
= & \left\|y_{k}-x^{*}\right\|^{2}-2 \lambda\left\langle y_{k}-x^{*}, v_{k+1}\right\rangle+\frac{1}{\alpha_{1}} \lambda\left\|v_{k+1}\right\|^{2}-\lambda\left(\frac{1}{\alpha_{1}}-1-\lambda\right)\left\|v_{k+1}\right\|^{2} \\
\leq & \left\|y_{k}-x^{*}\right\|^{2}-2 \lambda\left\langle y_{k}-x^{*}, v_{k+1}\right\rangle+2 \lambda\left\langle y_{k}-x^{*}+(1-\lambda) v_{k}, v_{k+1}\right\rangle \\
& -\lambda\left(\frac{1}{\alpha_{1}}-1-\lambda\right)\left\|v_{k+1}\right\|^{2} \\
= & \left\|y_{k}-x^{*}\right\|^{2}+2 \lambda(1-\lambda)\left\langle v_{k}, v_{k+1}\right\rangle-\lambda\left(\frac{1}{\alpha_{1}}-1-\lambda\right)\left\|v_{k+1}\right\|^{2} .
\end{aligned}
$$

Since

$$
\lambda(1-\lambda)\left\|v_{k+1}-v_{k}\right\|^{2}=\lambda(1-\lambda)\left(\left\|v_{k+1}\right\|^{2}-2\left\langle v_{k+1}, v_{k}\right\rangle+\left\|v_{k}\right\|^{2}\right)
$$


we obtain

$$
\begin{aligned}
& \left\|x_{k+1}-x^{*}\right\|^{2}+\lambda\left\|v_{k+1}\right\|^{2} \\
\leq & \left\|y_{k}-x^{*}\right\|^{2}+\lambda(1-\lambda)\left\|v_{k}\right\|^{2}-\lambda(1-\lambda)\left\|v_{k+1}-v_{k}\right\|^{2}-\lambda\left(\frac{1}{\alpha_{1}}-2\right)\left\|v_{k+1}\right\|^{2} .
\end{aligned}
$$

It follows from

$$
\begin{aligned}
\left\langle x_{k}-x^{*}, A^{*}(I-T) A x_{k}\right\rangle & =\left\langle A x_{k}-A x^{*},(I-T) A x_{k}-(I-T) A x^{*}\right\rangle \\
& \geq \frac{1}{2 \alpha}\left\|(I-T) A x_{k}-(I-T) A x^{*}\right\|^{2} \\
& =\frac{1}{2 \alpha_{2}}\left\|(I-T) A x_{k}\right\|^{2}
\end{aligned}
$$

that

$$
\begin{aligned}
& \left\|y_{k}-x^{*}\right\|^{2} \\
= & \left\|x_{k}-\gamma_{k} A^{*}(I-T) A x_{k}-x^{*}\right\|^{2} \\
\leq & \left\|x_{k}-x^{*}\right\|^{2}-2 \gamma_{k}\left\langle x_{k}-x^{*}, A^{*}(I-T) A x_{k}\right\rangle+\gamma_{k}^{2}\|A\|^{2}\left\|(I-T) A x_{k}\right\|^{2} \\
\leq & \left\|x_{k}-x^{*}\right\|^{2}-\frac{1}{\alpha_{2}} \gamma_{k}\left\|(I-T) A x_{k}\right\|^{2}+\gamma_{k}^{2}\|A\|^{2}\left\|(I-T) A x_{k}\right\|^{2} \\
= & \left\|x_{k}-x^{*}\right\|^{2}-\gamma_{k}\left(\frac{1}{\alpha_{2}}-\gamma_{k}\|A\|^{2}\right)\left\|(I-T) A x_{k}\right\|^{2} .
\end{aligned}
$$

Hence, we obtain

$$
\begin{aligned}
& \left\|x_{k+1}-x^{*}\right\|^{2}+\lambda\left\|v_{k+1}\right\|^{2} \\
\leq & \left\|x_{k}-x^{*}\right\|^{2}-\gamma_{k}\left(\frac{1}{\alpha_{2}}-\gamma_{k}\|A\|^{2}\right)\left\|(I-T) A x_{k}\right\|^{2}+\lambda(1-\lambda)\left\|v_{k}\right\|^{2} \\
& -\lambda(1-\lambda)\left\|v_{k+1}-v_{k}\right\|^{2}-\lambda\left(\frac{1}{\alpha_{1}}-2\right)\left\|v_{k+1}\right\|^{2} \\
= & \left\|x_{k}-x^{*}\right\|^{2}+\lambda\left\|v_{k}\right\|^{2}-\gamma_{k}\left(\frac{1}{\alpha_{2}}-\gamma_{k}\|A\|^{2}\right)\left\|(I-T) A x_{k}\right\|^{2}-\lambda^{2}\left\|v_{k}\right\|^{2} \\
& -\lambda(1-\lambda)\left\|v_{k+1}-v_{k}\right\|^{2}-\lambda\left(\frac{1}{\alpha_{1}}-2\right)\left\|v_{k+1}\right\|^{2} .
\end{aligned}
$$

Let $s_{k}=\left\|x_{k}-x^{*}\right\|^{2}+\lambda\left\|v_{k}\right\|^{2}$. By the assumptions on $\gamma_{k}, \lambda, \alpha_{1}$ and (3.9), we can get $s_{k+1} \leq s_{k}$, which implies that the sequence $\left\{s_{k}\right\}$ is non-increasing and lower bounded by 0 and so $\lim _{k \rightarrow \infty} s_{k}$ exists. Thus it follows that $\left\{s_{k}\right\}$ is bounded and hence $\left\{x_{k}\right\}$ is bounded. Moreover, from (3.9), we also have

$$
\gamma_{k}\left(\frac{1}{\alpha_{2}}-\gamma_{k}\|A\|^{2}\right)\left\|(I-T) A x_{k}\right\|^{2}+\lambda^{2}\left\|v_{k}\right\|^{2} \leq s_{k}-s_{k+1},
$$

which implies that

$$
\lim _{k \rightarrow \infty}\left\|(I-T) A x_{k}\right\|=0
$$

and

$$
\lim _{k \rightarrow \infty}\left\|v_{k}\right\|=0 .
$$


So, $\lim _{k \rightarrow \infty}\left\|x_{k}-x^{*}\right\|^{2}=\lim _{k \rightarrow \infty}\left(s_{k}-\lambda\left\|v_{k}\right\|^{2}\right)=\lim _{k \rightarrow \infty} s_{k}$ exists. By the iterative algorithm (3.1), we have

$$
\begin{aligned}
& \left\|x_{k}-U x_{k}\right\| \\
= & \| x_{k}-y_{k}-(1-\lambda) v_{k}+y_{k}+(1-\lambda) v_{k} \\
& -U\left(y_{k}+(1-\lambda) v_{k}\right)+U\left(y_{k}+(1-\lambda) v_{k}\right)-U x_{k} \| \\
\leq & \left\|x_{k}-y_{k}-(1-\lambda) v_{k}\right\|+\left\|y_{k}+(1-\lambda) v_{k}-U\left(y_{k}+(1-\lambda) v_{k}\right)\right\| \\
& +\left\|U\left(y_{k}+(1-\lambda) v_{k}\right)-U x_{k}\right\| \\
\leq & 2\left\|x_{k}-y_{k}-(1-\lambda) v_{k}\right\|+\left\|y_{k}+(1-\lambda) v_{k}-U\left(y_{k}+(1-\lambda) v_{k}\right)\right\| \\
\leq & 2\left\|x_{k}-y_{k}\right\|+2\left\|(1-\lambda) v_{k}\right\|+\left\|v_{k+1}\right\| \\
= & 2\left\|\gamma_{k} A^{*}(I-T) A x_{k}\right\|+2(1-\lambda)\left\|v_{k}\right\|+\left\|v_{k+1}\right\| .
\end{aligned}
$$

From the conditions on $\gamma_{k}, \lambda$ and (3.10)-(3.12), it follows that

$$
\left\|x_{k}-U x_{k}\right\| \rightarrow 0
$$

as $k \rightarrow \infty$.

Next, we prove that $\omega_{w}\left(x_{k}\right) \subseteq \Gamma$. Assume that $\bar{x} \in \omega_{w}\left(x_{k}\right)$, i.e., there exist a subsequence $\left\{x_{k_{j}}\right\}$ of $\left\{x_{k}\right\}$ such that $x_{k_{j}} \rightarrow \bar{x}$. Then we have $A x_{k_{j}} \rightarrow A \bar{x}$. It follows from (3.10), (3.13) and Lemma 2.1 that $\bar{x} \in F(U)$ and $A \bar{x} \in F(T)$, which implies that $\bar{x} \in \Gamma$. So $\omega_{w}\left(x_{k}\right) \subseteq \Gamma$ is proved.

Finally, by Lemma 2.2, we have $x_{k} \rightarrow x^{*}$, where $x^{*}$ is a solution of the SCFP (1.1). Thus, it follows from $v_{k} \rightarrow 0$ that $\left(v_{k}, x_{k}\right) \rightarrow\left(0, x^{*}\right)$. This completes the proof.

It is well known that a firmly nonexpansive operator is $\frac{1}{2}$-averaged and so, from Theorem 3.1, we can deduce easily the following:

Corollary 3.1. Let $U$ and $T$ be firmly nonexpansive operators. Suppose that $0<\lambda \leq 1$ and

$$
0<\liminf _{k \rightarrow \infty} \gamma_{k} \leq \limsup _{k \rightarrow \infty} \gamma_{k}<\frac{2}{\|A\|^{2}} .
$$

Let $\left\{\left(v_{k}, x_{k}\right)\right\}$ be the sequence generated by

$$
\left\{\begin{array}{l}
x_{0}, v_{0} \in H_{1} \text { chosen arbitrarily, } \\
y_{k}=x_{k}-\gamma_{k} A^{*}(I-T) A x_{k} \\
v_{k+1}=(I-U)\left(y_{k}+(1-\lambda) v_{k}\right), \\
x_{k+1}=y_{k}-\lambda v_{k+1}
\end{array}\right.
$$

for each $k \geq 0$. Then the sequence $\left\{x_{k}\right\}$ converges weakly to a point $x^{*}$, where $x^{*} \in \Gamma$, and the sequence $\left\{\left(v_{k}, x_{k}\right)\right\}$ weakly converges to the point $\left(0, x^{*}\right)$.

Remark 3.1. In Theorem 3.1, the averaged operators $U$ and $T$ can be replaced by the directed operators which satisfy that $I-U$ and $I-T$ are demiclosed at the origin. This does not affect the convergence result because of

$$
\|(I-U) x\|^{2}=\left\|(I-U) x-(I-U) x^{*}\right\|^{2} \leq\left\langle(I-U) x, x-x^{*}\right\rangle
$$

for all $x \in H$ and $x^{*} \in F(U)$, where $U$ is a directed operator. 
Remark 3.2. When $\lambda=1$, algorithm (3.1) becomes CQ-algorithm (1.5) for solving the SCFP:

$$
x_{k+1}=U\left(x_{k}-\gamma_{k} A^{*}(I-T) A x_{k}\right)
$$

for each $k \geq 0$. Thus Theorem 3.1 extends the related results of Censor and Segal [4] for solving the SCFP. Moreover, taking $U=P_{C}$ and $T=P_{Q}$, algorithm (3.1) becomes CQ-algorithm (1.4), which was proposed by Byrne [3] for solving the SFP.

\section{Strong CONVERGENCE THEOREMS}

In this section, we modify algorithm (3.1) to show that the algorithm has the strongly convergence. It is known that the viscosity approximation method is often used to approximate a fixed point of a nonexpansive mapping $U$ in Hilbert spaces, which it is defined as follows [14]:

$$
x_{k+1}=\beta_{k} f\left(x_{k}\right)+\left(1-\beta_{k}\right) U\left(x_{k}\right)
$$

for each $k \geq 1$, where $\left\{\beta_{k}\right\} \subseteq[0,1]$ and $f$ is a contraction.

Now, we use method (4.1) to get our strong convergence resutls.

Theorem 4.1. Let $f: H_{1} \rightarrow H_{1}$ be a $\rho$-contraction mapping. Let $U$ be an $\alpha_{1}$-averaged mapping and let $T$ be an $\alpha_{2}$-averaged mapping. If the sequences $\left\{\beta_{k}\right\} \subset[0,1],\left\{\gamma_{k}\right\}$ and the constants $\lambda, \alpha_{1}, \rho$ satisfy the following conditions:

(a) $\beta_{k} \rightarrow 0$ as $k \rightarrow \infty$;

(b) $\sum_{k=0}^{\infty} \beta_{k}=\infty$;

(c) $0 \leq \rho<\frac{1}{\sqrt{2}}$ and $0<\liminf _{k \rightarrow \infty} \gamma_{k} \leq \limsup _{k \rightarrow \infty} \gamma_{k}<\frac{1}{\alpha_{2}\|A\|^{2}}$;

(d) $0<\lambda \leq 1,0<\alpha_{1}<\frac{1}{2}$ or $0<\lambda<1,0<\alpha_{1} \leq \frac{1}{2}$,

then the sequence $\left\{\left(v_{k}, x_{k}\right)\right\}$ generated by the algorithm:

$$
\left\{\begin{array}{l}
x_{0}, v_{0} \in H_{1} \text { chosen arbitrarily, } \\
y_{k}=x_{k}-\gamma_{k} A^{*}(I-T) A x_{k} \\
\bar{v}_{k}=(I-U)\left(y_{k}+(1-\lambda) v_{k}\right) \\
\bar{x}_{k}=y_{k}-\lambda \bar{v}_{k} \\
v_{k+1}=\beta_{k} f\left(v_{k}\right)+\left(1-\beta_{k}\right) \bar{v}_{k} \\
x_{k+1}=\beta_{k} f\left(x_{k}\right)+\left(1-\beta_{k}\right) \bar{x}_{k}
\end{array}\right.
$$

for each $k \geq 0$ converges strongly to $\left(0, x^{*}\right)$, where $x^{*}$ is a solution of the SCFP (1.1) which solves the variational inequality problem:

$$
\left\langle(I-f) x^{*}, x-x^{*}\right\rangle \geq 0
$$

for any $x \in \Gamma$.

Proof. Let $x^{*} \in \Gamma$ be a solution of (4.3). Then $x^{*} \in F(U)$ and $A x^{*} \in F(T)$. From (3.9), it follows that

$$
\begin{aligned}
& \left\|\bar{x}_{k}-x^{*}\right\|^{2}+\lambda\left\|\bar{v}_{k}\right\|^{2} \\
\leq & \left\|x_{k}-x^{*}\right\|^{2}+\lambda\left\|v_{k}\right\|^{2}-\gamma_{k}\left(\frac{1}{\alpha_{2}}-\gamma_{k}\|A\|^{2}\right)\left\|(I-T) A x_{k}\right\|^{2}-\lambda^{2}\left\|v_{k}\right\|^{2} \\
& -\lambda(1-\lambda)\left\|\bar{v}_{k}-v_{k}\right\|^{2}-\lambda\left(\frac{1}{\alpha_{1}}-2\right)\left\|\bar{v}_{k}\right\|^{2} .
\end{aligned}
$$


In particular, we have

$$
\left\|\bar{x}_{k}-x^{*}\right\|^{2}+\lambda\left\|\bar{v}_{k}\right\|^{2} \leq\left\|x_{k}-x^{*}\right\|^{2}+\lambda\left\|v_{k}\right\|^{2}
$$

Thus it follows that

$$
\begin{aligned}
& \left\|x_{k+1}-x^{*}\right\|^{2}+\lambda\left\|v_{k+1}\right\|^{2} \\
= & \left\|\beta_{k} f\left(x_{k}\right)+\left(1-\beta_{k}\right) \bar{x}_{k}-x^{*}\right\|^{2}+\lambda\left\|\beta_{k} f\left(v_{k}\right)+\left(1-\beta_{k}\right) \bar{v}_{k}\right\|^{2} \\
\leq & \beta_{k}\left\|f\left(x_{k}\right)-x^{*}\right\|^{2}+\left(1-\beta_{k}\right)\left\|\bar{x}_{k}-x^{*}\right\|^{2}+\lambda\left(\beta_{k}\left\|f\left(v_{k}\right)\right\|^{2}+\left(1-\beta_{k}\right)\left\|\bar{v}_{k}\right\|^{2}\right) \\
\leq & 2 \beta_{k}\left(\left\|f\left(x_{k}\right)-f\left(x^{*}\right)\right\|^{2}+\left\|f\left(x^{*}\right)-x^{*}\right\|^{2}\right)+\left(1-\beta_{k}\right)\left\|\bar{x}_{k}-x^{*}\right\|^{2} \\
& +\lambda\left[2 \beta_{k}\left(\left\|f\left(v_{k}\right)-f(0)\right\|^{2}+\|f(0)\|^{2}\right)+\left(1-\beta_{k}\right)\left\|\bar{v}_{k}\right\|^{2}\right] \\
\leq & 2 \beta_{k} \rho^{2}\left\|x_{k}-x^{*}\right\|^{2}+2 \beta_{k}\left\|f\left(x^{*}\right)-x^{*}\right\|^{2}+\left(1-\beta_{k}\right)\left\|\bar{x}_{k}-x^{*}\right\|^{2} \\
& +2 \lambda \beta_{k} \rho^{2}\left\|v_{k}\right\|^{2}+2 \lambda \beta_{k}\|f(0)\|^{2}+\lambda\left(1-\beta_{k}\right)\left\|\bar{v}_{k}\right\|^{2} \\
\leq & \left(1-\beta_{k}\right)\left(\left\|x_{k}-x^{*}\right\|^{2}+\lambda\left\|v_{k}\right\|^{2}\right)+2 \beta_{k} \rho^{2}\left(\left\|x_{k}-x^{*}\right\|^{2}+\lambda\left\|v_{k}\right\|^{2}\right)+2 \beta_{k}\left(\left\|f\left(x^{*}\right)-x^{*}\right\|^{2}+\lambda\|f(0)\|^{2}\right) \\
= & {\left[1-\beta_{k}\left(1-2 \rho^{2}\right)\right]\left(\left\|x_{k}-x^{*}\right\|^{2}+\lambda\left\|v_{k}\right\|^{2}\right)+\beta_{k}\left(1-2 \rho^{2}\right) \frac{2}{1-2 \rho^{2}}\left(\left\|f\left(x^{*}\right)-x^{*}\right\|^{2}+\lambda\|f(0)\|^{2}\right) . }
\end{aligned}
$$

Setting $s_{k}=\left\|x_{k}-x^{*}\right\|^{2}+\lambda\left\|v_{k}\right\|^{2}$, we have

$$
s_{k+1} \leq\left[1-\beta_{k}\left(1-2 \rho^{2}\right)\right] s_{k}+\beta_{k}\left(1-2 \rho^{2}\right) \frac{2}{1-2 \rho^{2}}\left(\left\|f\left(x^{*}\right)-x^{*}\right\|^{2}+\lambda\|f(0)\|^{2}\right) .
$$

It follows from induction that

$$
s_{k} \leq \max \left\{s_{0}, \frac{2}{1-2 \rho^{2}}\left(\left\|f\left(x^{*}\right)-x^{*}\right\|^{2}+\lambda\|f(0)\|^{2}\right)\right\}
$$

for each $k \geq 0$, which implies that $\left\{x_{k}\right\}$ and $\left\{v_{k}\right\}$ are bounded. Also, $\left\{\bar{x}_{k}\right\},\left\{\bar{v}_{k}\right\},\left\{f\left(x_{k}\right)\right\}$ and $\left\{f\left(v_{k}\right)\right\}$ are bounded. From (4.2), we have

$$
\begin{aligned}
& \left\|x_{k+1}-x^{*}\right\|^{2}+\lambda\left\|v_{k+1}\right\|^{2} \\
= & \left\|\beta_{k} f\left(x_{k}\right)+\left(1-\beta_{k}\right) \bar{x}_{k}-x^{*}\right\|^{2}+\lambda\left\|\beta_{k} f\left(v_{k}\right)+\left(1-\beta_{k}\right) \bar{v}_{k}\right\|^{2} \\
= & \beta_{k}^{2}\left\|f\left(x_{k}\right)-x^{*}\right\|^{2}+2 \beta_{k}\left(1-\beta_{k}\right)\left\langle f\left(x_{k}\right)-x^{*}, \bar{x}_{k}-x^{*}\right\rangle+\left(1-\beta_{k}\right)^{2}\left\|\bar{x}_{k}-x^{*}\right\|^{2} \\
& +\lambda\left(\beta_{k}^{2}\left\|f\left(v_{k}\right)\right\|^{2}+2 \beta_{k}\left(1-\beta_{k}\right)\left\langle f\left(v_{k}\right), \bar{v}_{k}\right\rangle+\left(1-\beta_{k}\right)^{2}\left\|\bar{v}_{k}\right\|^{2}\right) \\
= & \beta_{k}^{2}\left\|f\left(x_{k}\right)-x^{*}\right\|^{2}+2 \beta_{k}\left(1-\beta_{k}\right)\left(\left\langle f\left(x_{k}\right)-f\left(x^{*}\right), \bar{x}_{k}-x^{*}\right\rangle\right. \\
& \left.+\left\langle f\left(x^{*}\right)-x^{*}, \bar{x}_{k}-x^{*}\right\rangle\right)+\left(1-\beta_{k}\right)^{2}\left\|\bar{x}_{k}-x^{*}\right\|^{2}+\lambda \beta_{k}^{2}\left\|f\left(v_{k}\right)\right\|^{2} \\
& +2 \lambda \beta_{k}\left(1-\beta_{k}\right)\left(\left\langle f\left(v_{k}\right)-f(0), \bar{v}_{k}\right\rangle+\left\langle f(0), \bar{v}_{k}\right\rangle\right)+\lambda\left(1-\beta_{k}\right)^{2}\left\|\bar{v}_{k}\right\|^{2} \\
\leq & \beta_{k}^{2}\left\|f\left(x_{k}\right)-x^{*}\right\|^{2}+\beta_{k}\left(1-\beta_{k}\right)\left(\left\|f\left(x_{k}\right)-f\left(x^{*}\right)\right\|^{2}+\left\|\bar{x}_{k}-x^{*}\right\|^{2}\right) \\
& +2 \beta_{k}\left(1-\beta_{k}\right)\left\langle f\left(x^{*}\right)-x^{*}, \bar{x}_{k}-x^{*}\right\rangle+\left(1-\beta_{k}\right)^{2}\left\|\bar{x}_{k}-x^{*}\right\|^{2}+\lambda \beta_{k}^{2}\left\|f\left(v_{k}\right)\right\|^{2} \\
& +\lambda \beta_{k}\left(1-\beta_{k}\right)\left(\left\|f\left(v_{k}\right)-f(0)\right\|^{2}+\left\|\bar{v}_{k}\right\|^{2}\right) \\
& +2 \lambda \beta_{k}\left(1-\beta_{k}\right)\left\langle f(0), \bar{v}_{k}\right\rangle+\lambda\left(1-\beta_{k}\right)^{2}\left\|\bar{v}_{k}\right\|^{2} \\
\leq & \beta_{k}^{2}\left\|f\left(x_{k}\right)-x^{*}\right\|^{2}+\beta_{k}\left(1-\beta_{k}\right) \rho^{2}\left\|x_{k}-x^{*}\right\|^{2}+\left(1-\beta_{k}\right)\left\|\bar{x}_{k}-x^{*}\right\|^{2} \\
& +2 \beta_{k}\left(1-\beta_{k}\right)\left\langle f\left(x^{*}\right)-x^{*}, \bar{x}_{k}-x^{*}\right\rangle+\lambda \beta_{k}^{2}\left\|f\left(v_{k}\right)\right\|^{2} \\
& +\lambda \beta_{k}\left(1-\beta_{k}\right) \rho^{2}\left\|v_{k}\right\|^{2}+\lambda\left(1-\beta_{k}\right)\left\|\bar{v}_{k}\right\|^{2}+2 \lambda \beta_{k}\left(1-\beta_{k}\right)\left\langle f(0), \bar{v}_{k}\right\rangle .
\end{aligned}
$$


So, by (4.5) and (4.7), we have

$$
\begin{aligned}
s_{k+1} \leq & {\left[1-\beta_{k}\left(1-\left(1-\beta_{k}\right) \rho^{2}\right)\right] s_{k}+\beta_{k}\left[\beta_{k}\left(\left\|f\left(x_{k}\right)-x^{*}\right\|^{2}+\lambda\left\|f\left(v_{k}\right)\right\|^{2}\right)\right.} \\
& \left.+2\left(1-\beta_{k}\right)\left(\left\langle f\left(x^{*}\right)-x^{*}, \bar{x}_{k}-x^{*}\right\rangle+\lambda\left\langle f(0), \bar{v}_{k}\right\rangle\right)\right] \\
= & \left(1-\lambda_{k}\right) s_{k}+\lambda_{k} \delta_{k},
\end{aligned}
$$

where

$$
\lambda_{k}=\beta_{k}\left(1-\left(1-\beta_{k}\right) \rho^{2}\right)
$$

and

$$
\begin{aligned}
\delta_{k}= & \frac{2\left(1-\beta_{k}\right)\left(\left\langle f\left(x^{*}\right)-x^{*}, \bar{x}_{k}-x^{*}\right\rangle+\lambda\left\langle f(0), \bar{v}_{k}\right\rangle\right)}{1-\left(1-\beta_{k}\right) \rho^{2}} \\
& +\frac{\beta_{k}\left(\left\|f\left(x_{k}\right)-x^{*}\right\|^{2}+\lambda\left\|f\left(v_{k}\right)\right\|^{2}\right)}{1-\left(1-\beta_{k}\right) \rho^{2}} .
\end{aligned}
$$

On the other hand, from (4.4) and (4.7), it follows that

$$
\begin{aligned}
s_{k+1} \leq & {\left[1-\beta_{k}\left(1-\left(1-\beta_{k}\right) \rho^{2}\right)\right] s_{k}+\beta_{k}^{2}\left(\left\|f\left(x_{k}\right)-x^{*}\right\|^{2}+\lambda\left\|f\left(v_{k}\right)\right\|^{2}\right) } \\
& +2 \beta_{k}\left(1-\beta_{k}\right)\left(\left\langle f\left(x^{*}\right)-x^{*}, \bar{x}_{k}-x^{*}\right\rangle+\lambda\left\langle f(0), \bar{v}_{k}\right\rangle\right) \\
& -\left(1-\beta_{k}\right)\left[\gamma_{k}\left(\frac{1}{\alpha_{2}}-\gamma_{k}\|A\|^{2}\right)\left\|(I-T) A x_{k}\right\|^{2}+\lambda^{2}\left\|v_{k}\right\|^{2}\right. \\
& \left.+\lambda(1-\lambda)\left\|\bar{v}_{k}-v_{k}\right\|^{2}+\lambda\left(\frac{1}{\alpha_{1}}-2\right)\left\|\bar{v}_{k}\right\|^{2}\right] \\
\leq & s_{k}+\beta_{k}^{2}\left(\left\|f\left(x_{k}\right)-x^{*}\right\|^{2}+\lambda\left\|f\left(v_{k}\right)\right\|^{2}\right) \\
& +2 \beta_{k}\left(1-\beta_{k}\right)\left(\left\langle f\left(x^{*}\right)-x^{*}, \bar{x}_{k}-x^{*}\right\rangle+\lambda\left\langle f(0), \bar{v}_{k}\right\rangle\right) \\
& -\left(1-\beta_{k}\right)\left[\gamma_{k}\left(\frac{1}{\alpha_{2}}-\gamma_{k}\|A\|^{2}\right)\left\|(I-T) A x_{k}\right\|^{2}+\lambda^{2}\left\|v_{k}\right\|^{2}\right. \\
& \left.+\lambda(1-\lambda)\left\|\bar{v}_{k}-v_{k}\right\|^{2}+\lambda\left(\frac{1}{\alpha_{1}}-2\right)\left\|\bar{v}_{k}\right\|^{2}\right] .
\end{aligned}
$$

Now, by setting

$$
\begin{aligned}
\mu_{k}= & \beta_{k}^{2}\left(\left\|f\left(x_{k}\right)-x^{*}\right\|^{2}+\lambda\left\|f\left(v_{k}\right)\right\|^{2}\right) \\
& +2 \beta_{k}\left(1-\beta_{k}\right)\left(\left\langle f\left(x^{*}\right)-x^{*}, \bar{x}_{k}-x^{*}\right\rangle+\lambda\left\langle f(0), \bar{v}_{k}\right\rangle\right)
\end{aligned}
$$

and

$$
\begin{aligned}
\eta_{k}= & \left(1-\beta_{k}\right)\left[\gamma_{k}\left(\frac{1}{\alpha_{2}}-\gamma_{k}\|A\|^{2}\right)\left\|(I-T) A x_{k}\right\|^{2}+\lambda^{2}\left\|v_{k}\right\|^{2}\right. \\
& \left.+\lambda(1-\lambda)\left\|\bar{v}_{k}-v_{k}\right\|^{2}+\lambda\left(\frac{1}{\alpha_{1}}-2\right)\left\|\bar{v}_{k}\right\|^{2}\right],
\end{aligned}
$$

one sees that (4.9) can be rewritten as the following form

$$
s_{k+1} \leq s_{k}-\eta_{k}+\mu_{k}
$$

for each $k \geq 0$. By the assumptions on $\left\{\beta_{k}\right\}$ and $\rho$, we have

$$
\sum_{k=0}^{\infty} \lambda_{k}=\infty, \quad \lim _{k \rightarrow \infty} \mu_{k}=0,
$$

from the boundedness of $\left\{x_{k}\right\}$ and $\left\{v_{k}\right\}$. To use Lemma 2.3, it suffices to verify that, for all subsequence $\left\{k_{l}\right\} \subset\{k\}, \lim _{l \rightarrow \infty} \eta_{k_{l}}=0$ implies

$$
\limsup _{l \rightarrow \infty} \delta_{k_{l}} \leq 0
$$


Thus, since $\lim _{l \rightarrow \infty} \eta_{k_{l}}=0$, we have

$$
\lim _{l \rightarrow \infty}\left[\gamma_{k_{l}}\left(\frac{1}{\alpha_{2}}-\gamma_{k_{l}}\|A\|^{2}\right)\left\|(I-T) A x_{k_{l}}\right\|^{2}+\lambda^{2}\left\|v_{k_{l}}\right\|^{2}+\lambda(1-\lambda)\left\|\bar{v}_{k_{l}}-v_{k_{l}}\right\|^{2}+\lambda\left(\frac{1}{\alpha_{1}}-2\right)\left\|\bar{v}_{k_{l}}\right\|^{2}\right]=0 .
$$

From the assumptions on $\alpha_{1}, \alpha_{2}, \lambda$ and $\left\{\gamma_{k}\right\}$, we obtain

$$
\begin{gathered}
\lim _{l \rightarrow \infty}\left\|(I-T) A x_{k_{l}}\right\|=0, \\
\lim _{l \rightarrow \infty}\left\|v_{k_{l}}\right\|=0
\end{gathered}
$$

and

$$
\lim _{l \rightarrow \infty}\left\|\bar{v}_{k_{l}}\right\|=0 .
$$

In a similar way as in Theorem 3.1, we can get $\omega_{w}\left(x_{k_{l}}\right) \subseteq \Gamma$. In addition, from (4.13), (4.15) and

$$
\begin{aligned}
\left\|x_{k_{l}}-\bar{x}_{k_{l}}\right\| & =\left\|x_{k_{l}}-x_{k_{l}}+\gamma_{k_{l}} A^{*}(I-T) A x_{k_{l}}+\lambda \bar{v}_{k_{l}}\right\| \\
& =\left\|\gamma_{k_{l}} A^{*}(I-T) A x_{k_{l}}+\lambda \bar{v}_{k_{l}}\right\| \\
& \leq \gamma_{k_{l}}\|A\|\left\|(I-T) A x_{k_{l}}\right\|+\lambda\left\|\bar{v}_{k_{l}}\right\|,
\end{aligned}
$$

we obtain

$$
\lim _{l \rightarrow \infty}\left\|x_{k_{l}}-\bar{x}_{k_{l}}\right\|=0
$$

Note that

$$
\lim _{l \rightarrow \infty}\left(1-\left(1-\beta_{k_{l}}\right) \rho^{2}\right)=1-\rho^{2}
$$

and

$$
\lim _{l \rightarrow \infty} \beta_{k_{l}}\left(\left\|f\left(x_{k_{l}}\right)-x^{*}\right\|^{2}+\lambda\left\|f\left(v_{k_{l}}\right)\right\|^{2}\right)=0 .
$$

To get (4.11), we only need to verify

$$
\limsup _{l \rightarrow \infty}\left(\left\langle f\left(x^{*}\right)-x^{*}, \bar{x}_{k_{l}}-x^{*}\right\rangle+\lambda\left\langle f(0), \bar{v}_{k_{l}}\right\rangle\right) \leq 0 .
$$

From (4.16), we can take subsequence $\left\{\left(v_{k_{l_{j}}}, x_{k_{l_{j}}}\right)\right\}$ of $\left\{\left(v_{k_{l}}, x_{k_{l}}\right)\right\}$ such that $x_{k_{l_{j}}} \rightarrow \widetilde{x}$ as $j \rightarrow \infty$ and

$$
\begin{aligned}
& \limsup _{l \rightarrow \infty}\left(\left\langle f\left(x^{*}\right)-x^{*}, \bar{x}_{k_{l}}-x^{*}\right\rangle+\lambda\left\langle f(0), \bar{v}_{k_{l}}\right\rangle\right) \\
= & \lim _{j \rightarrow \infty}\left(\left\langle f\left(x^{*}\right)-x^{*}, \bar{x}_{k_{l_{j}}}-x^{*}\right\rangle+\lambda\left\langle f(0), \bar{v}_{k_{l_{j}}}\right\rangle\right) \\
= & \lim _{j \rightarrow \infty}\left\langle f\left(x^{*}\right)-x^{*}, x_{k_{l_{j}}}-x^{*}\right\rangle \\
= & \left\langle f\left(x^{*}\right)-x^{*}, \widetilde{x}-x^{*}\right\rangle .
\end{aligned}
$$

Since $\omega_{w}\left(x_{k_{l}}\right) \subset \Gamma$ and $x^{*}$ is a solution of (4.3), it follows from (4.17) that

$$
\limsup _{l \rightarrow \infty}\left(\left\langle f\left(x^{*}\right)-x^{*}, \bar{x}_{k_{l}}-x^{*}\right\rangle+\lambda\left\langle f(0), \bar{v}_{k_{l}}\right\rangle\right) \leq 0 .
$$

It follows from Lemma 2.3 that

$$
\lim _{k \rightarrow \infty} s_{k}=\lim _{k \rightarrow \infty}\left(\left\|x_{k}-x^{*}\right\|^{2}+\lambda\left\|v_{k}\right\|^{2}\right)=0,
$$

which implies that $x_{k} \rightarrow x^{*}, v_{k} \rightarrow 0$ and $\left(v_{k}, x_{k}\right) \rightarrow\left(0, x^{*}\right)$, where $x^{*}$ is a solution of the SCFP (1.1), which solves (4.3). This completes the proof.

From Theorem 4.1, we can easily deduce the following results. 
Corollary 4.1. Let $f: H_{1} \rightarrow H_{1}$ be a $\rho$-contraction mapping. Let $U$ and $T$ be directed operators. Assume that $I-U$ and $I-T$ are demiclosed at the origin. If the sequences $\left\{\beta_{k}\right\} \subset[0,1],\left\{\gamma_{k}\right\}$ and the constants $\lambda, \rho$ satisfy the following conditions:

(a) $\beta_{k} \rightarrow 0$ as $k \rightarrow \infty$;

(b) $\sum_{k=0}^{\infty} \beta_{k}=\infty$;

(c) $0 \leq \rho<\frac{1}{\sqrt{2}}$ and $0<\liminf _{k \rightarrow \infty} \gamma_{k} \leq \limsup _{k \rightarrow \infty} \gamma_{k}<\frac{2}{\|A\|^{2}}$;

(d) $0<\lambda<1$,

then the sequence $\left\{\left(v_{k}, x_{k}\right)\right\}$ generated by the algorithm:

$$
\left\{\begin{array}{l}
x_{0}, v_{0} \in H_{1} \text { chosen arbitrarily, } \\
y_{k}=x_{k}-\gamma_{k} A^{*}(I-T) A x_{k} \\
\bar{v}_{k}=(I-U)\left(y_{k}+(1-\lambda) v_{k}\right), \\
\bar{x}_{k}=y_{k}-\lambda \bar{v}_{k} \\
v_{k+1}=\beta_{k} f\left(v_{k}\right)+\left(1-\beta_{k}\right) \bar{v}_{k} \\
x_{k+1}=\beta_{k} f\left(x_{k}\right)+\left(1-\beta_{k}\right) \bar{x}_{k}
\end{array}\right.
$$

for each $k \geq 0$, converges strongly to $\left(0, x^{*}\right)$, where $x^{*}$ is a solution of the SCFP (1.1) which solves the variational inequality problem $\left\langle(I-f) x^{*}, x-x^{*}\right\rangle \geq 0, \forall x \in \Gamma$.

Corollary 4.2. Let $f: H_{1} \rightarrow H_{1}$ be a $\rho$-contraction mapping. Let $U$ be an $\alpha_{1}$-averaged mapping and let $T$ be an $\alpha_{2}$-averaged mapping. If the sequences $\left\{\beta_{k}\right\} \subset[0,1],\left\{\gamma_{k}\right\}$ and the constants $\alpha_{1}, \rho$ satisfy the following conditions:

(a) $\beta_{k} \rightarrow 0$ as $k \rightarrow \infty$;

(b) $\sum_{k=0}^{\infty} \beta_{k}=\infty$;

(c) $0 \leq \rho<\frac{1}{\sqrt{2}}$ and $0<\liminf _{k \rightarrow \infty} \gamma_{k} \leq \limsup _{k \rightarrow \infty} \gamma_{k}<\frac{1}{\alpha_{2}\|A\|^{2}}$;

(d) $0<\alpha_{1}<\frac{1}{2}$,

then the sequence $\left\{x_{k}\right\}$ generated by the algorithm:

$$
\left\{\begin{array}{l}
x_{0} \in H_{1} \text { chosen arbitrarily, } \\
y_{k}=x_{k}-\gamma_{k} A^{*}(I-T) A x_{k}, \\
x_{k+1}=\beta_{k} f\left(x_{k}\right)+\left(1-\beta_{k}\right) U\left(y_{k}\right)
\end{array}\right.
$$

for each $k \geq 0$, converges strongly to $x^{*}$, where $x^{*}$ is a solution of the SCFP (1.1) which solves the variational inequality problem $\left\langle(I-f) x^{*}, x-x^{*}\right\rangle \geq 0, \forall x \in \Gamma$.

\section{NUMERICAL EXPERIMENTS}

In this section, we present some preliminary numerical results and show the efficiency of our proposed methods to solve the SFP (1.2). All the codes are written in MATLAB and are performed on a personal Lenovo computer with Pentium(R) Dual-Core CPU @ 2.4GHz and RAM 2.00GB.

For the sake of convenience, we denote the vector with all elements 0 by $e_{0}$ and the vector with all elements 1 by $e_{1}$ in what follows. That is, $e_{0}=(0,0, \cdots, 0)^{T}$ and $e_{1}=(1,1, \cdots, 1)^{T}$. The rand $n$ is a MATLAB command to generate normally distributed random numbers. 
TABLE 1. Numerical results for solving Example 5.1 with initial point $\left(10 e_{1},-10 e_{1}\right)$.

\begin{tabular}{|c|c|c|c|c|c|c|c|c|c|c|c|}
\hline & & & Initial & point & $x_{0}=10 e_{1}$ & $v_{0}=-10 e_{1}$ & & & & & \\
\hline & $\lambda$ & 0.1 & 0.2 & 0.3 & 0.4 & 0.5 & 0.6 & 0.7 & 0.8 & 0.9 & 1 \\
\hline$N=10$ & $k$ & 10 & 10 & 10 & 71 & 72 & 71 & 71 & 70 & 71 & 71 \\
\hline$N=20$ & $k$ & 168 & 178 & 167 & 179 & 179 & 14 & 177 & 162 & 161 & 177 \\
\hline$N=40$ & $k$ & 1555 & 1455 & 1548 & 1309 & 1505 & 1318 & 1300 & 1286 & 382 & 1256 \\
\hline
\end{tabular}

TABLE 2. Numerical results for solving Example 5.1 with initial point $\left(20 e_{1},-5 e_{1}\right)$.

\begin{tabular}{|c|c|c|c|c|c|c|c|c|c|c|c|}
\hline & & & Initial & point & $x_{0}=20 e_{1}$ & $v_{0}=-5 e_{1}$ & & & & & \\
\hline & $\lambda$ & 0.1 & 0.2 & 0.3 & 0.4 & 0.5 & 0.6 & 0.7 & 0.8 & 0.9 & 1 \\
\hline$N=10$ & $k$ & 109 & 97 & 70 & 70 & 70 & 70 & 66 & 66 & 66 & 146 \\
\hline$N=20$ & $k$ & 139 & 22 & 161 & 140 & 164 & 164 & 137 & 138 & 163 & 157 \\
\hline$N=40$ & $k$ & 1432 & 1418 & 1267 & 1338 & 873 & 1279 & 245 & 827 & 234 & 345 \\
\hline
\end{tabular}

Now, we compare algorithm (3.1) with the CQ algorithm proposed by Byrne [3] to solve Example 5.1 .

Example 5.1. We consider the SFP (1.2) as follows: the matrix $A=\left(a_{i, j}\right)_{N \times N}$ and $a_{i, j} \in(0,10)$ are generated randomly, the nonempty closed convex sets $C$ and $Q$ are

$$
C=\left\{x \in R^{N}:\|x\| \leq 40\right\}
$$

and

$$
Q=\left\{y \in R^{N}: L \leq y \leq M\right\},
$$

where $L$ and $M$ are the lower and upper bounds of the box $Q, L \in\left[10 e_{1}, 40 e_{1}\right]$ and $M \in\left[50 e_{1}, 100 e_{1}\right]$ are all generated randomly. Since the projection operators $P_{C}$ and $P_{Q}$ are $\frac{1}{2}$-averaged operators, it follows that $\alpha_{1}=\alpha_{2}=\frac{1}{2}$ in Theorem 3.1. In this part, we take the step-size $\gamma_{k}=\frac{1.95}{\|A\|^{2}}$ and

$$
p(x)=\left\|x-P_{C} x\right\|+\left\|A x-P_{Q}(A x)\right\|<\varepsilon=10^{-8}
$$

as the stopping criterion. Since the parameter $\lambda \in(0,1]$ is very important for dual variable, we try different values of $\lambda$ to solve Example 5.1. When the parameter $\lambda=1$, the algorithm generated by (3.1) becomes the exactly CQ-algorithm proposed by Byrne [3]. We showed the comparison of the algorithm generated by (3.1) with the exactly CQ-algorithm proposed by Byrne [3] for solving Example 5.1 with different initial points and different dimensions $N$. We have the numerical results in table 1 and table 2, where we denote the number of iterations by $k$. We can see from table 1 and table 2 that the algorithm (3.1) with suitable $\lambda$ converges faster than the exactly CQ-algorithm $(\lambda=1)$.

\section{Acknowledgements}

This project was supported by the Theoretical and Computational Science (TaCS) Center under Computational and Applied Science for Smart Innovation Cluster (CLASSIC), Faculty of Science, KMUTT. Moreover, Poom Kumam was supported by the Thailand Research Fund (TRF) and the King Mongkut's 
University of Technology Thonburi (KMUTT) under the TRF Research Scholar Award (Grant No. RSA6080047).

\section{REFERENCES}

[1] F.E. Browder, Nonlinear operators and nonlinear equations of evolution in Banach spaces, Proc. Sympos. Pure Math., vol. 18 (part 2), Amer. Math. Soc., Providence, RI (1976).

[2] C. Byrne, A unified treatment of some iterative algorithms in signal processing and image reconstruction, Inverse Probl. 20 (2004), 103-120.

[3] C. Byrne, Iterative oblique projection onto convex subsets and the split feasibility problem, Inverse Prob. 18 (2002), 441-453.

[4] Y. Censor, A. Segal, The split common fixed point problem for directed operators, J. Convex Anal. 16 (2009), $587-600$.

[5] Y. Censor, T. Elfving, A multiprojection algorithm using Bregman projections in a product space, Numer. Algorithms 8 (1994), 221-239.

[6] Y. Censor, T. Bortfeld, B. Martin, A. Trofimov, A unified approach for inversion problems in intensity modulated radiation therapy, Phys. Med. Biol. 51 (2006), 2353-2365.

[7] Y. Censor, A. Gibalin, S. Reich, Algorithms for the split variational inequality problem, Numer. Algorithms 59 (2012), 301-323.

[8] S.S. Chang, L. Wang, Y. Zhao, On a class of split equality fixed point problems in Hilbert spaces, J. Nonlinear Var. Anal. 1 (2017), 201-212.

[9] H. Cui, F. Wang, Iterative methods for the split commom fixed point problem in Hilbert spaces, Fixed Point Theory Appl. 2014 (2014), Article ID 78.

[10] P. Chen, J. Huang, X. Zhang, A primal-dual fixed point algorithm for convex separable minimization with applications to image restoration, Inverse Probl. 29 (2013), Article ID 025011.

[11] J. Gornicki, Weak convergence theorems for asymptotically nonexpansive mappings in uniformly convex Banach spaces, Comment. Math. Univ. Carolin. 301 (1998), 249-252.

[12] S. He, C. Yang, Solving the variational inequality problem defined on intersection of finite level sets, Abstr. Appl. Anal.2013 (2013), Article ID 942315.

[13] E. Masad, S. Reich, A note on the multiple-set split convex feasibility problem in Hilbert space, J. Nonlinear Convex Anal. 8 (2007), 367-371.

[14] A. Moudafi, Viscosity approxmation methods for fixed points problems, J. Math. Anal. Appl. 241 (2000), 46-55.

[15] A. Moudafi, A note on the split common fixed point problem for quasi-nonexpansive operators, Nonlinear Anal. 74 (2011), 4083-4087.

[16] A. Moudafi, The split common fixed point problem for demicontractive mappings, Inverse Probl. 26 (2010), Article ID 055007.

[17] Y.C. Tang, J.G. Peng, L.W. Liu, A cyclic algorithm for the split common fixed point problem of demicontractive mappings in Hilbert spaces, Math. Model. Anal. 17 (2012), 457-466.

[18] U. Witthayarat, Afrah A.N. Abdou, Y.J. Cho, Shrinking projection methods for solving split equilibrium problems and fixed point problems for asymptotically nonexpansive in Hilbert spaces, Fixed Point Theory Appl. 2015 (2015), Article ID 200 .

[19] J. Zhao, S. He, Viscosity approximation methods for split common fixed-point problem of directed operators, Numer. Funct. Anal. Optim. 36 (2015), 528-547. 